\title{
In vivo characterization of abnormalities in small-bowel diseases using probe-based confocal laser endomicroscopy ${ }^{1}$
}

\section{다)(웅}

\author{
Authors \\ Shibata', Tetsuya Tsukamoto², Makoto Kuroda² \\ Institutions \\ 1 Department of Gastroenterology, Fujita Health \\ University School of Medicine, Toyoake, Aichi, Japan \\ 2 Department of Diagnostic Pathology I, Fujita Health \\ University School of Medicine, Toyoake, Aichi, Japan
}

Naoki Ohmiya ${ }^{1}$, Noriyuki Horiguchi ${ }^{1}$, Tomomitsu Tahara ${ }^{1}$, Mitsuo Nagasaka ${ }^{1}$, Yoshihito Nakagawa ${ }^{1}$, Tomoyuki

submitted 8.8.2016

accepted after revision 13.2 .2017

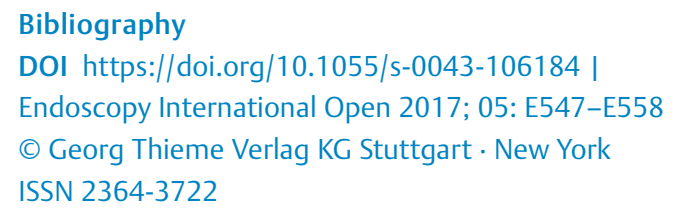

Corresponding author

Naoki Ohmiya, MD PhD, Department of Gastroenterology, Fujita Health University School of Medicine, 1-98 Kutsukake-cho, Toyoake, Aichi 470-1192, Japan

Fax: +81-562-938300

nohmiya@med.nagoya-u.ac.jp

\section{ABSTRACT}

Background and study aims Probe-based confocal laser endomicroscopy ( $p C L E$ ) enables real-time optical biopsy. Little is known about pCLE imaging deep inside the small bowel, therefore the aim of this study was to determine its usefulness.

Patients and methods Between April 2014 and January 2016, we performed 38 pCLE examinations during double- balloon enteroscopy with intravenous fluorescein in 37 patients with: tumors $(n=10)$, vascular disorders $(n=6)$, inflammatory diseases and drug injuries $(n=13)$, other disorders $(n=4)$, and normal findings $(n=4)$. We measured the calibers of capillary and lymphatic vessels at 15 different sites and compared the calibers between pCLE images and histopathology. We also compared PCLE findings with pathologic diagnosis.

Results The inner diameters of capillary vessels beneath the epithelium and in the middle of villi were $16.2 \pm 3.0 \mu \mathrm{m}$ and $14.5 \pm 3.1 \mu \mathrm{m}$, respectively, in the PCLE images, but these were not consistent with formalin-fixed paraffin-embedded histology. In tumors, larger capillary vessels were observed in aggressive malignant lymphoma and metastasis, and a "soccer ball-like pattern" constituting homogenous dark cells packed with polygonal, narrower capillary vessels was characteristic in PCLE images of a malignant lymphoma follicle. Hereditary hemorrhagic telangiectasia and angiodysplasia contained anastomosis of capillary vessels of different calibers. In IgA vasculitis, segmental capillary strictures were observed. Intestinal lymphangiectasia with protein-losing enteropathy contained a reticular network of lymphatic vessels and dilated lymphatic ducts accompanied by numerous cell gaps. pCLE findings corresponded to pathologic diagnosis in 32 examinations (91\%). Conclusions pCLE is useful for in vivo analysis of abnormalities of the capillary and lymphatic vessels and epithelium, and for diagnosis in various small-bowel diseases.

Study registration: UMIN 000013857

\section{Introduction}

Confocal laser endomicroscopy (CLE) is a novel endoscopic imaging technique that provides in vivo histology during ongoing endoscopy. Two CLE systems have been developed for the gastrointestinal tract: an endoscope-integrated system and a

\footnotetext{
1 A poster including part of this material was presented at the American Society for Gastrointestinal Endoscopy (DDW 2015 Washington D.C.) on 18 May 2015.
}

probe-based system; however, the endoscope-integrated system is inaccessible deep within the small bowel and is no longer commercially available. The probe-based system was developed by Mauna Kea Technologies (Cellvizio; Mauna Kea Technologies, Paris, France) [1]. This miniprobe can be introduced through the working channel of any endoscope to visualize the tissue at a microscopic level at the same time as the macroscopic imaging [2]. Furthermore, the GastroFlex UHD and Coloflex UHD probes are $3 \mathrm{~m}$ and $4 \mathrm{~m}$ long, respectively, and therefore they can be used during balloon-assisted enteroscopy. To 
our knowledge, little is known about the application of CLE in small-bowel diseases [3]. These miniprobes provide a resolution of up to $1 \mu \mathrm{m}$ after the administration of fluorescent contrast, depth of focus between 55 and $65 \mu \mathrm{m}$, and a fast videorate scanning of 12 images per second. CLE may enable more live images than conventional pathology.

In this study, we determined the roles of probe-based CLE (pCLE) during double-balloon enteroscopy (DBE) for the detection of abnormalities and the diagnosis of various small-bowel diseases.

\section{Methods}

\section{Study design}

This was a retrospective single-center observational open-label pilot study. We measured the calibers of capillary and lymphatic vessels between pCLE images and histopathology, and determined the vessel characteristics in various small-bowel diseases. Next, we retrospectively compared the pCLE findings with pathological findings, and determined their concordance rate. This study was reviewed and approved by the Institutional Review Board of Fujita University School of Medicine. This study was registered with the University Hospital Medical Information Network (UMIN 000013857).

\section{Double-balloon enteroscopy and probe-based confocal laser endomicroscopy}

DBE (Fujifilm Corporation, Tokyo, Japan) was performed as described previously $[4,5]$. pCLE examinations were performed with a Coloflex UHD or a Gastroflex UHD probe through the working channel $(2.8 \mathrm{~mm}$ or $3.2 \mathrm{~mm}$ diameter) of an enteroscope (EN-450T or 580 T) after intravenous injection of 250 $500 \mathrm{mg}$ fluorescein $(100 \mathrm{mg} / \mathrm{mL}$, Fluorescite, Alcon Japan Ltd., Tokyo, Japan).

\section{Measurement of the capillary and lymphatic vessel calibers and interpretation of other images}

Fluorescein-enhanced capillary vessels were classified into two types: capillary vessels beneath the epithelial cells and in the middle of the villi. The calibers of these two vessels at five different sites were estimated at three locations on one capillary vessel in one image with "measure object dimension" software in the Cellvizio viewer (Mauna Kea Technologies). In brief, the reader placed a cursor at one end of the fluorescein-enhanced vessel and then moved the cursor to the other end of the shortest dimension at right angles to the duct axis, which indicated the intraluminal diameter of the vessel. If the caliber varied in diameter, the reader measured both the shortest and longest diameters ( $\mathbf{F i g . 1 a ) . ~ L y m p h a t i c ~ v e s s e l s ~ w e r e ~ d e s i g n a t e d ~ a s ~ a ~}$ reticular network of unenhanced dark branching bands, and their calibers were estimated as above ( $\triangleright$ Fig. 1b). Other image interpretations included cell gap, erosion, fluorescence enhancement of lamina propria, and dark tumor cells.

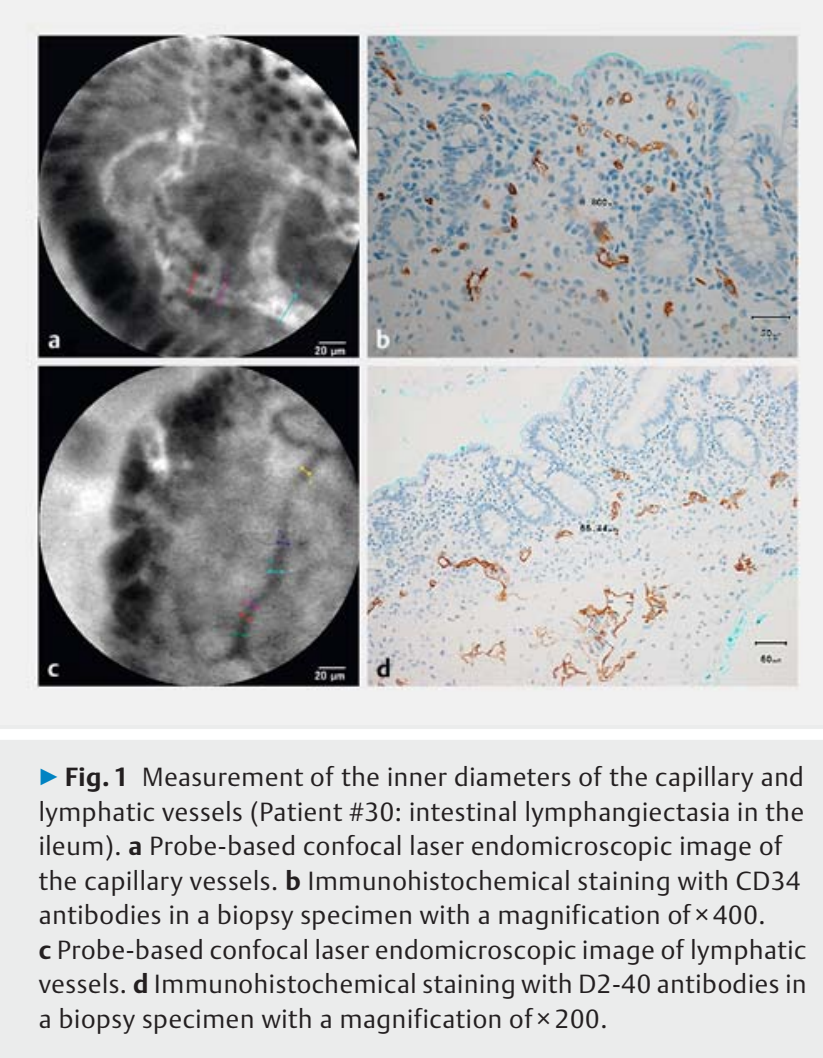

\section{Histologic analysis and comparison of the inner diameters of capillary vessels between PCLE and histology}

Biopsy specimens from DBE were fixed with $10 \%$ neutral buffered formalin, and paraffin-embedded sections were reviewed histologically by pathologists who were blinded to this study. Specific pathologic findings included dilated lymphatic lacteals in the lamina propria that were immunopositive with a monoclonal antibody against D2-40 (Dako, Tokyo, Japan) in intestinal lymphangiectasia; lymphoma cells that were immunopositive with monoclonal antibodies against CD20, CD10, bcl-2, and cyclinD1 (Dako), and crypt hyperplasia that was immunopositive with a monoclonal antibody Ki-67 (Dako) in villous atrophy.

In patients with dilated lymphatic lacteals that could be observed with $\mathrm{pCLE}$, bioptic specimens were immunostained with monoclonal antibodies against CD34 (Dako) and D2-40. The calibers of capillary vessels positive with CD34 at three different sites and lymphatic vessels positive with D2-40 at two different sites were measured with a point-to-point distance measuring function (Provis AX-80 optical microscope and DP20 digital camera, Olympus Corporation, Tokyo, Japan) and then were compared with those in the PCLE images.

\section{Statistical analysis}

The values presented in the text are expressed as means \pm standard deviation. Comparisons between lesions ( $n=15$ in each patient) and normal controls ( $n=60$ in four controls) were analyzed using the Mann-Whitney $U$ test. Comparisons of the calibers of capillary vessels positive with CD34 and lympha- 
tic vessels positive with D2-40 between immunostained bioptic specimens and pCLE images were analyzed using the MannWhitney $U$ test. Differences were considered statistically significant for $P$ values less than 0.05 .

\section{Results}

\section{Patients}

In total, 130 patients were admitted to Fujita Health University Hospital to undergo DBE between April 2014 and January 2016. Informed consent was obtained from 37 patients who underwent probe-based confocal laser endomicroscopy ( $p C L E$, Cellvizio; Mauna Kea Technologies, Paris, France) during DBE. The 37 patients underwent a total of 38 DBE and pCLE examinations; 24 via the oral route and 14 via the anal route using Coloflex UHD $(n=16)$ and Gastroflex UHD $(n=22)$ probes. Patient \#2 with follicular lymphoma underwent a second anal DBE examination 16 months after the first anal DBE examination, and rituximab monotherapy. Patient \#29 had villous atrophy and crypt apoptosis throughout the small intestine, and a few CD3-positive intraepithelial lymphocytes (5-10/100 epithelial cells). She was negative with anti-gliadin, anti-tissue transglutaminase, or anti-endomysial antibody. At colonoscopy, there were only several erosions in the rectum, but pathologically accompanied by a $25 \mu \mathrm{m}$ thick subepithelial collagen band. Her symptoms recovered and endoscopic findings improved with discontinuation of clonazepam, not with a gluten-free diet. Therefore, we diagnosed her as having clonazepam-induced intestinal injury.

Double-balloon enteroscopy was often in a torqued position, when advancing the probe through the working channel was difficult. In this case, we first put the top-down and/or left-right angle knobs to the neutral position. If impossible, we then withdrew the scope a little to loosen the scope angle. Clinical characteristics are presented in Table 1. The median number (range) of biopsy specimens at DBE in 31 patients was 4 (2 10). One polypectomy specimen, one endoscopic mucosal resection specimen, and one surgical specimen were taken from Patients \#10, \#11, and \#33, respectively. All authors had access to the study data, and reviewed and approved the final manuscript.

\section{Measurement of the inner diameter of capillary vessels and capillary characteristics at PCLE}

The inner diameters of capillary vessels beneath the epithelium and in the middle of the villi are shown in $>$ Table 2 . In four patients with an apparently normal small bowel with normal bioptic histology, the diameters of capillary vessels beneath the epithelium and in the middle of the villi (60 portions of capillary vessels each) were $16.2 \pm 3.0 \mu \mathrm{m}$ and $14.5 \pm 3.1 \mu \mathrm{m}$, respectively $(P=0.0009)$. Comparison between four normal controls and the inner diameters of capillary vessels beneath the epithelium or in the middle of the villi is shown in > Table 2 .

Throughout mantle cell lymphoma and follicular lymphoma, or in follicles adjacent to circumferential ulcers of diffuse large B-cell lymphoma (DLBCL), a "soccer ball-like pattern" was characteristic, with homogenous dark cells suggesting that lympho- ma cells (7 - 10 $\mu \mathrm{m}$ in diameter in small-cell lymphoma and 10 $20 \mu \mathrm{m}$ in diameter in large-cell lymphoma) were packed with polygonal, slightly narrow and stretched capillary vessels ( Fig.2a-d and $>$ Fig.3a,b). In lymphoid follicles with complete remission status, homogenous lymphoid-like cells were observed but not packed with those characteristic capillary vessels ( $\mathbf{F i g . 3 c , d ) . ~ I n ~ m e t a s t a s i s ~ f r o m ~ c e r v i c a l ~ c a n c e r , ~ i r r e g u l a r ~}$ dark nests suggesting metastatic squamous cell carcinoma were observed in the lamina propria, accompanied by markedly dilated capillary vessels suggesting tumor vessels ( $>$ Fig. $\mathbf{4 a - c}$ ). In a patient with hereditary hemorrhagic telangiectasia (HHT), dilated crooked capillary vessels were observed, and, notably, these vessels communicated with remarkably dilated vessels (30-40 $\mu \mathrm{m}$ in diameter) in the middle of the villi, suggesting a micro-arteriovenous malformation ( $\mathbf{F i g . 5 a - d ) . ~ I n ~ a ~ s o l i t a r y ~}$ angiodysplasia, various sizes of dilated proliferating capillary vessels appeared, anastomosing more frequently than normal ( Fig. 5e,f). In IgA vasculitis (formerly Henoch-Shöenlein purpura), multiple stenosing capillary vessels, like "a string of sausages", were observed around the ulcers, which may lead to ischemic changes ( $>$ Fig. $6 a-c)$.

\section{Measurement of the inner diameter of lymphatic vessels and lymphatic characteristics with PCLE}

Lymphatic vessels were observed in only five patients (17\%). In non-white type intestinal lymphangiectasias (Patients \#24 and \#26), CNSU (Patients \#16 and \#18), and metastasis from cervical cancer (Patient \#5), their inner diameters were $8.1 \pm 1.5 \mu \mathrm{m}$, $5.2 \pm 1.3 \mu \mathrm{m}, 4.8 \pm 1.0 \mu \mathrm{m}, 10.2 \pm 1.8 \mu \mathrm{m}$, and $6.8 \pm 1.3 \mu \mathrm{m}$, respectively.

In non-white type intestinal lymphangiectasia with proteinlosing enteropathy, a reticular network of thin dark branching bands was observed beneath the epithelium, and remarkably dilated unenhanced dark ducts deep in the mucosa, accompanied by fluorescence enhancement of lamina propria and numerous cell gaps ( $\triangleright$ Fig. $7 a-e)$.

\section{Preliminary comparison of the inner diameters of capillary vessels and lymphatic vessels between pCLE and histology}

In patients \#9, \#21, \#23, and \#30, the inner diameters of capillary vessels were $17.21 \pm 3.21,11.40 \pm 4.19,4.72 \pm 2.67$, and $7.47 \pm 1.56 \mu \mathrm{m}$, respectively, in bioptic specimens immunostained with CD34, which were different from those both beneath the epithelium and in the middle of villi in PCLE images $(24.2 \pm 13.1 \mu \mathrm{m}, P=0.01932,13.5 \pm 4.7 \mu \mathrm{m}, P=0.1824,14.2 \pm$ $4.6 \mu \mathrm{m}, P<0.0001,14.0 \pm 3.2 \mu \mathrm{m}, P<0.0001)$. Both the inner diameters of capillary vessels beneath the epithelium and in the middle of villi were counted up in PCLE images because their distinction was difficult in specimens that were immunostained with CD34.

In patients \#9, \#21, \#23, and \#30, the inner diameters of lymphatic vessels were $15.1 \pm 5.5,24.7 \pm 10.4,10.2 \pm 11.9$, and $27.1 \pm 17.0 \mu \mathrm{m}$, respectively, in bioptic specimens immunostained with D2-40, which were significantly different from those in PCLE images $(6.8 \pm 1.3 \mu \mathrm{m}, P=0.0157,4.8 \pm 1.0 \mu \mathrm{m}, P=$ $0.0005,10.2 \pm 1.8 \mu \mathrm{m}, P=0.5852$, and $8.1 \pm 1.5 \mu \mathrm{m}, P=0.0005)$. 


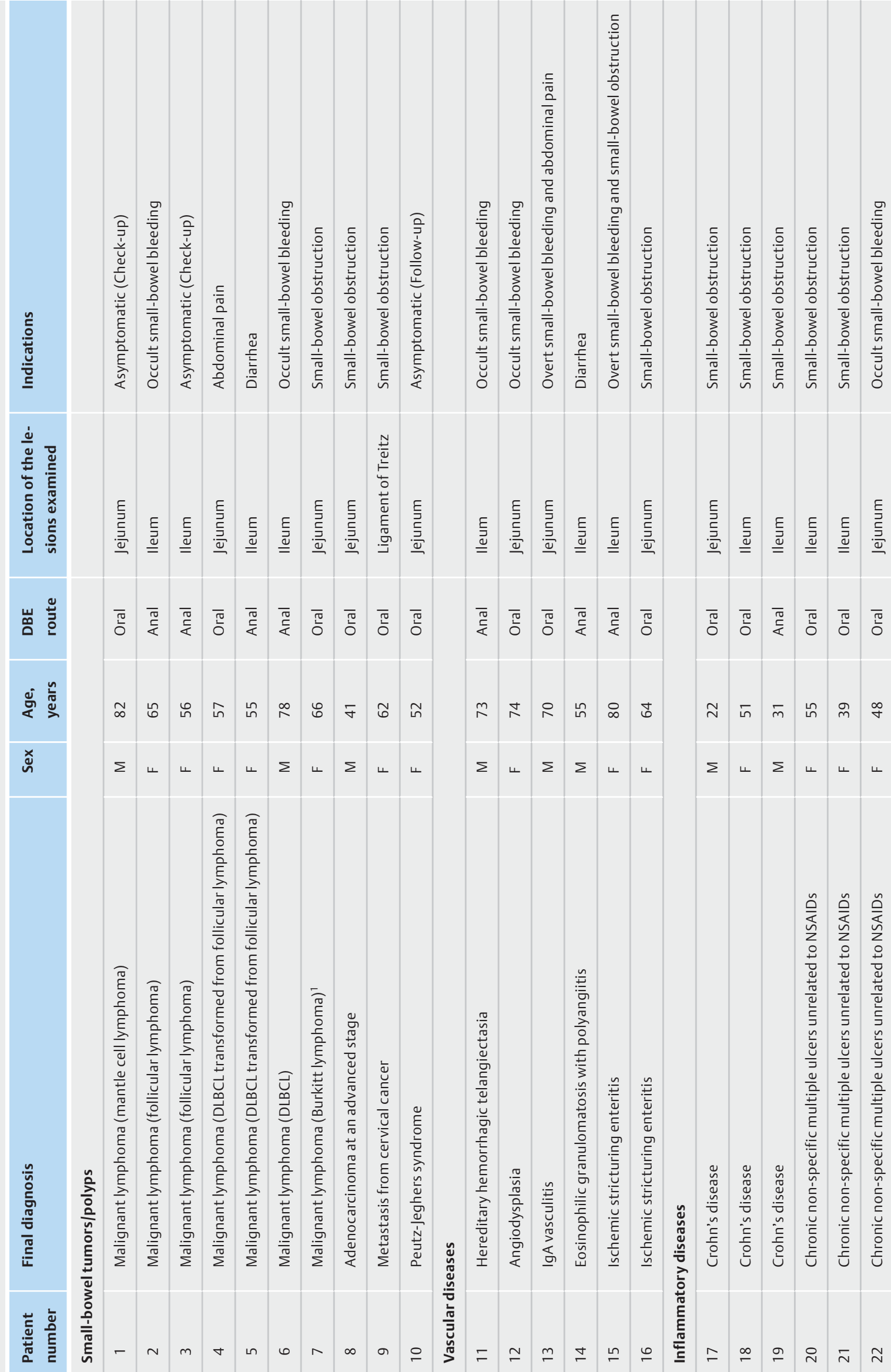




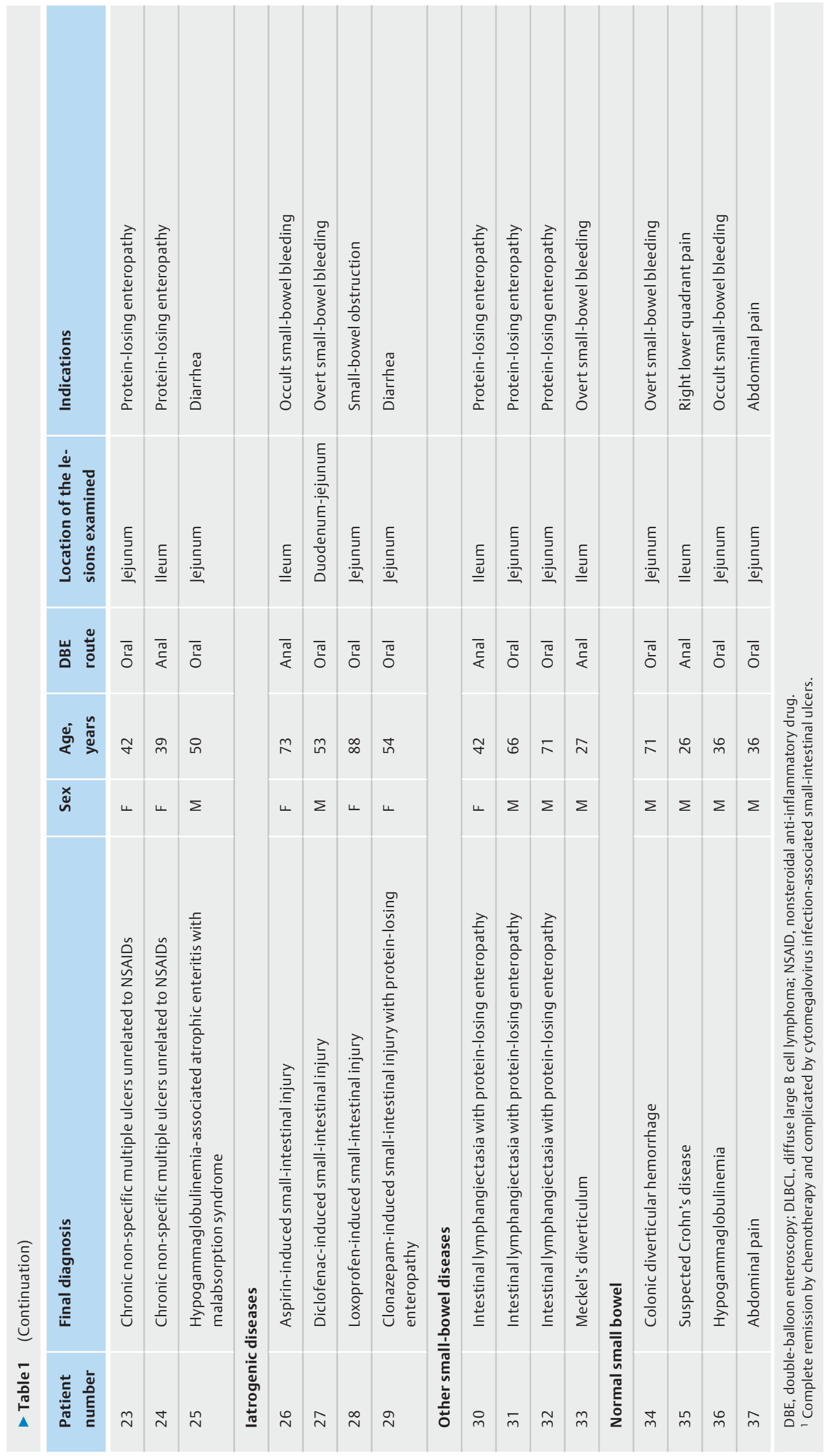




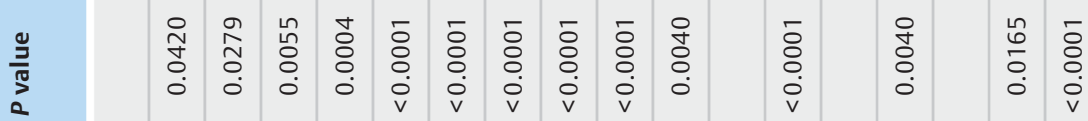

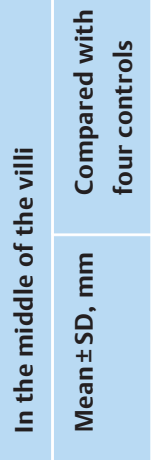

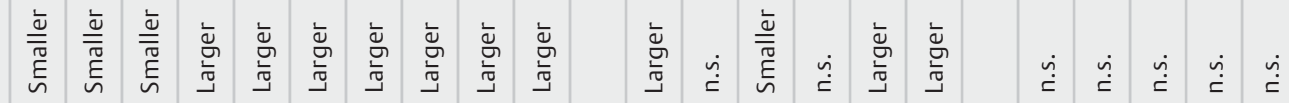

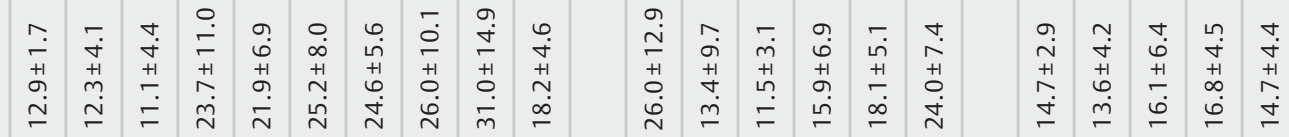

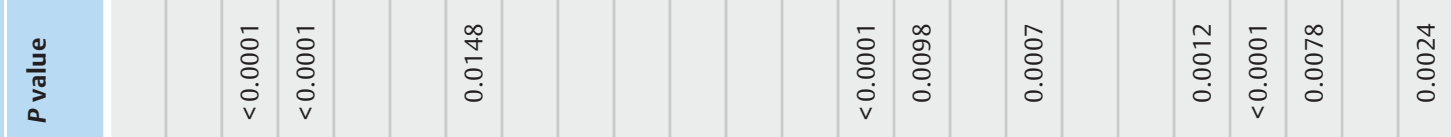

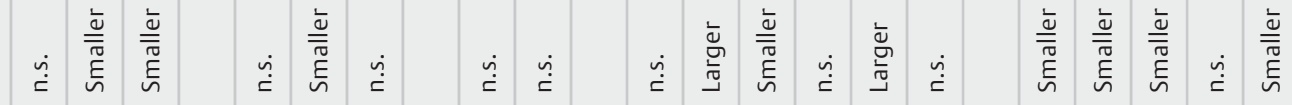

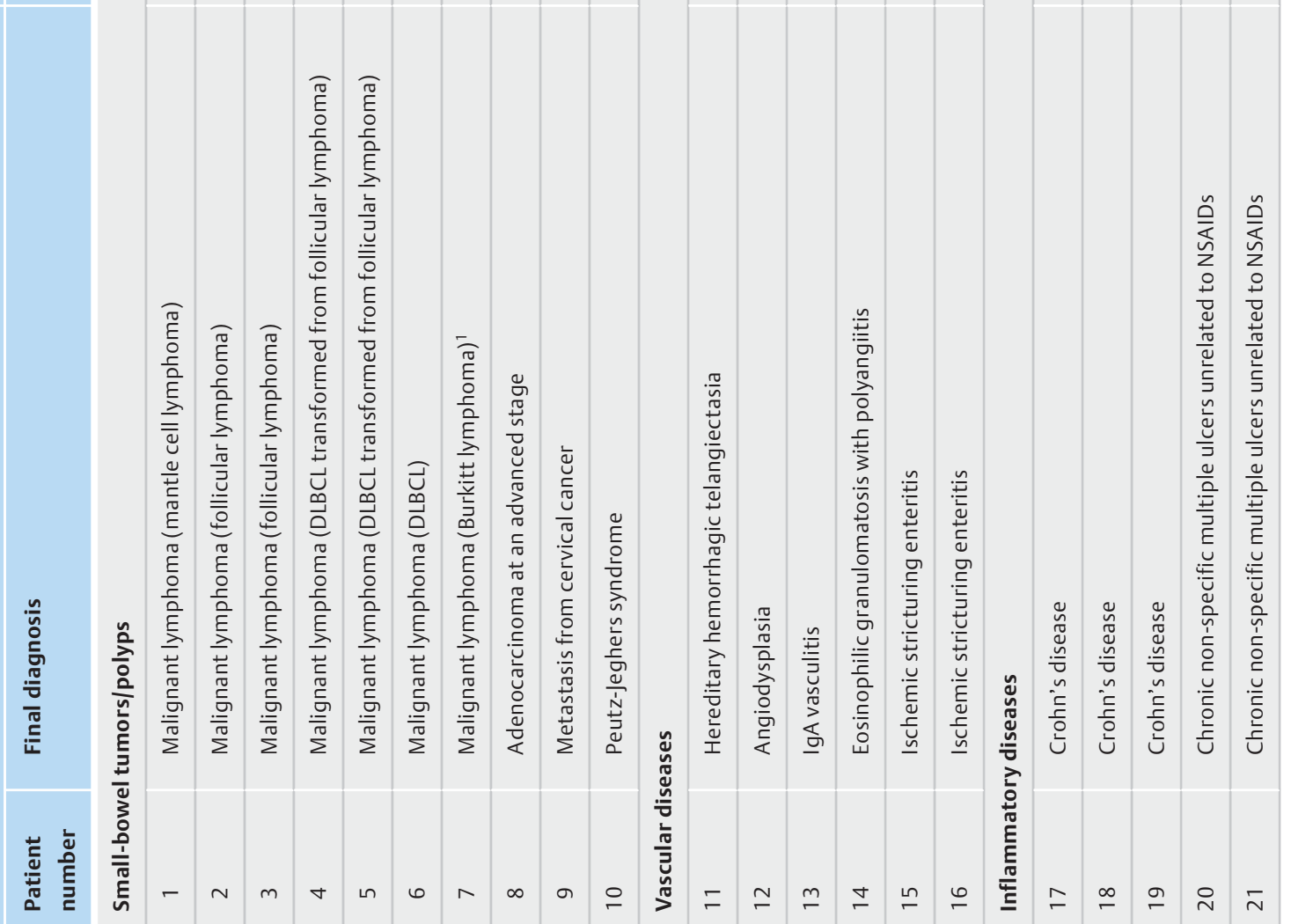




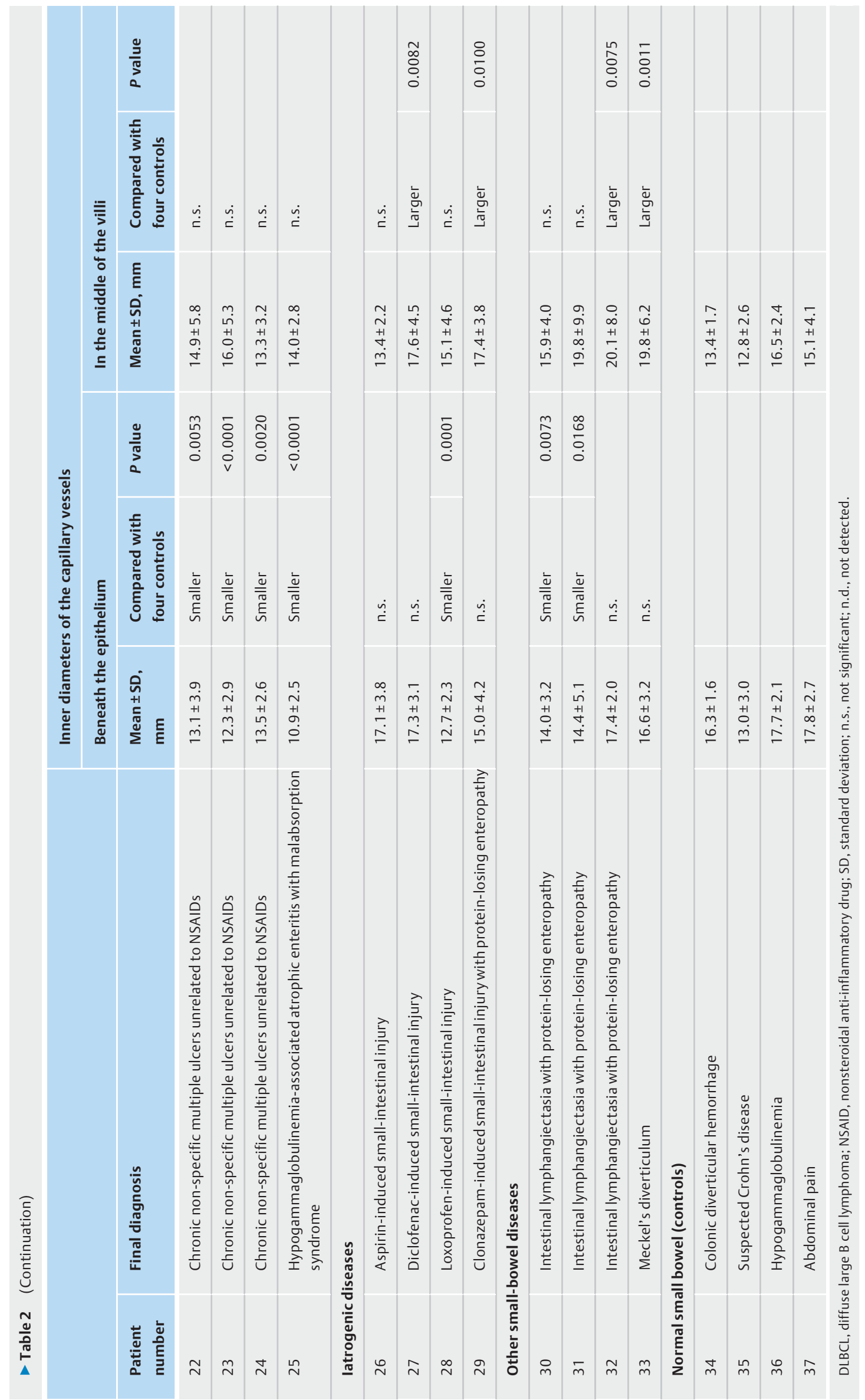



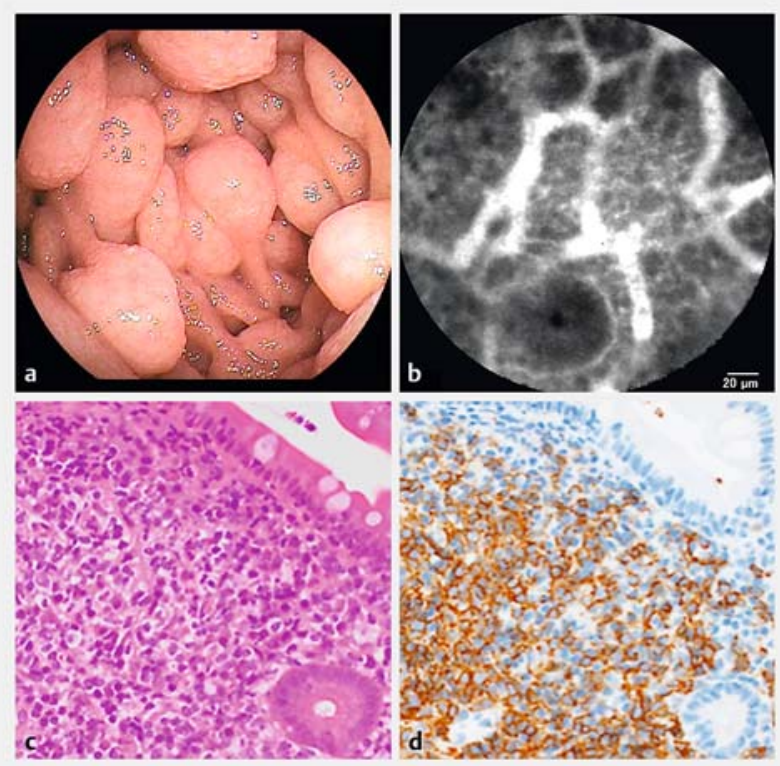

- Fig. 2 Mantle cell lymphoma (Patient \#1). a Enteroscopic view in the jejunum. b A probe-based confocal laser endomicroscopic image shows the homogenous cells packed with polygonal capillary vessels in a "soccer ball-like pattern. c H\&E staining in a biopsy specimen with a magnification of $\times 200$. $\mathbf{d}$ Immunohistochemical staining with CD20 antibodies in a biopsy specimen with a magnification of $\times 200$.
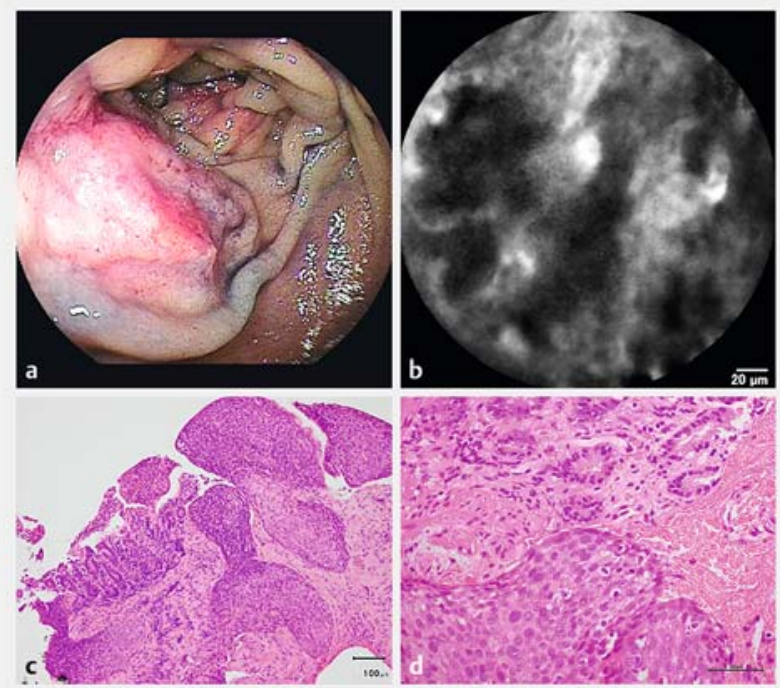

- Fig. 4 Metastasis from cervical cancer (Patient \#9). a Chromoenteroscopic view with indigo carmine after intravascular administration of fluorescein in the ligament of Treitz. $\mathbf{b}$ A probe-based confocal laser endomicroscopic image shows dark irregular tumor nests accompanied by dilated tortuous capillary vessels. $\mathrm{cH}$ HE staining in a biopsy specimen with a magnification of $\times 100$. $d \mathrm{H} \& \mathrm{E}$ staining in a biopsy specimen with a magnification of $\times 400$.
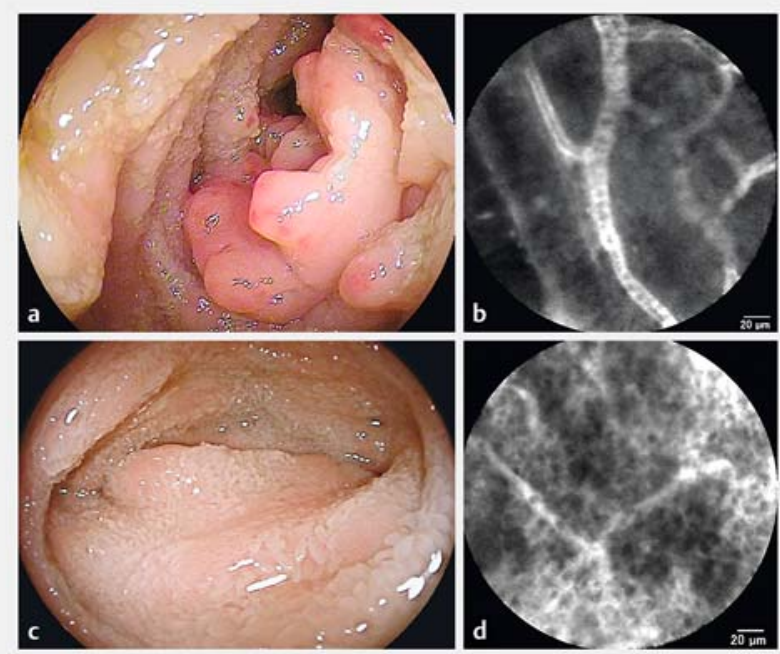

- Fig. 3 Follicular lymphoma (Patient \#2). a Enteroscopic view in the ileum before treatment. $\mathbf{b}$ A probe-based confocal laser endomicroscopic image shows the homogenous cells packed with polygonal capillary vessels in a "soccer ball-like pattern". c Enteroscopic view in the ileum after rituximab monotherapy. Slightly reddish follicles are observed. $\mathbf{d}$ A probe-based confocal laser endomicroscopic image does not show a "soccer ball-like pattern" after rituximab monotherapy.

\section{Epithelial and other pCLE findings}

In Peutz-Jeghers syndrome, a fluorescence-enhanced serrated homogenous epithelium was observed. In ischemic enteritis, numerous cell gaps were observed near the longitudinal ulcers and slightly dilated capillary vessels were frequently observed. Patients \#17-19 with Crohn's disease showed numerous cell gaps and fluorescence enhancement of the lamina propria. In chronic nonspecific multiple small-intestinal ulcers unrelated to NSAIDs (CNSU), narrow, angulated, and partly stenosing capillary vessels of various sizes were observed accompanied by numerous cell gaps and fluorescence enhancement of the lamina propria. In hypogammaglobulinemia-associated atrophic enteritis with malabsorption syndrome, villous atrophy and the base of the crypts appeared as roundish glands resembling healthy colonic daisy crypts. In diclofenac-induced small-bowel injury, fluorescence enhancement of the lamina propria and numerous cell gaps were observed, while they were much less frequently observed in aspirin-induced small-bowel injury. In clonazepam-induced enteropathy with protein-losing enteropathy, villous atrophy and the base of the crypts appeared as roundish glands resembling colonic daisy crypts.

\section{Comparison of pCLE findings with pathological diagnosis}

Of 35 pCLE examinations in 34 patients from whom biopsies, polypectomy, endoscopic mucosal resection, and surgical specimens were taken, pCLE findings in 32 (91\%) corresponded to pathologic diagnosis. The three inconsistent cases were patients \#7, \#32, and \#33. In patient \#7 with Burkitt lymphoma, complete remission was achieved by chemotherapy and no 

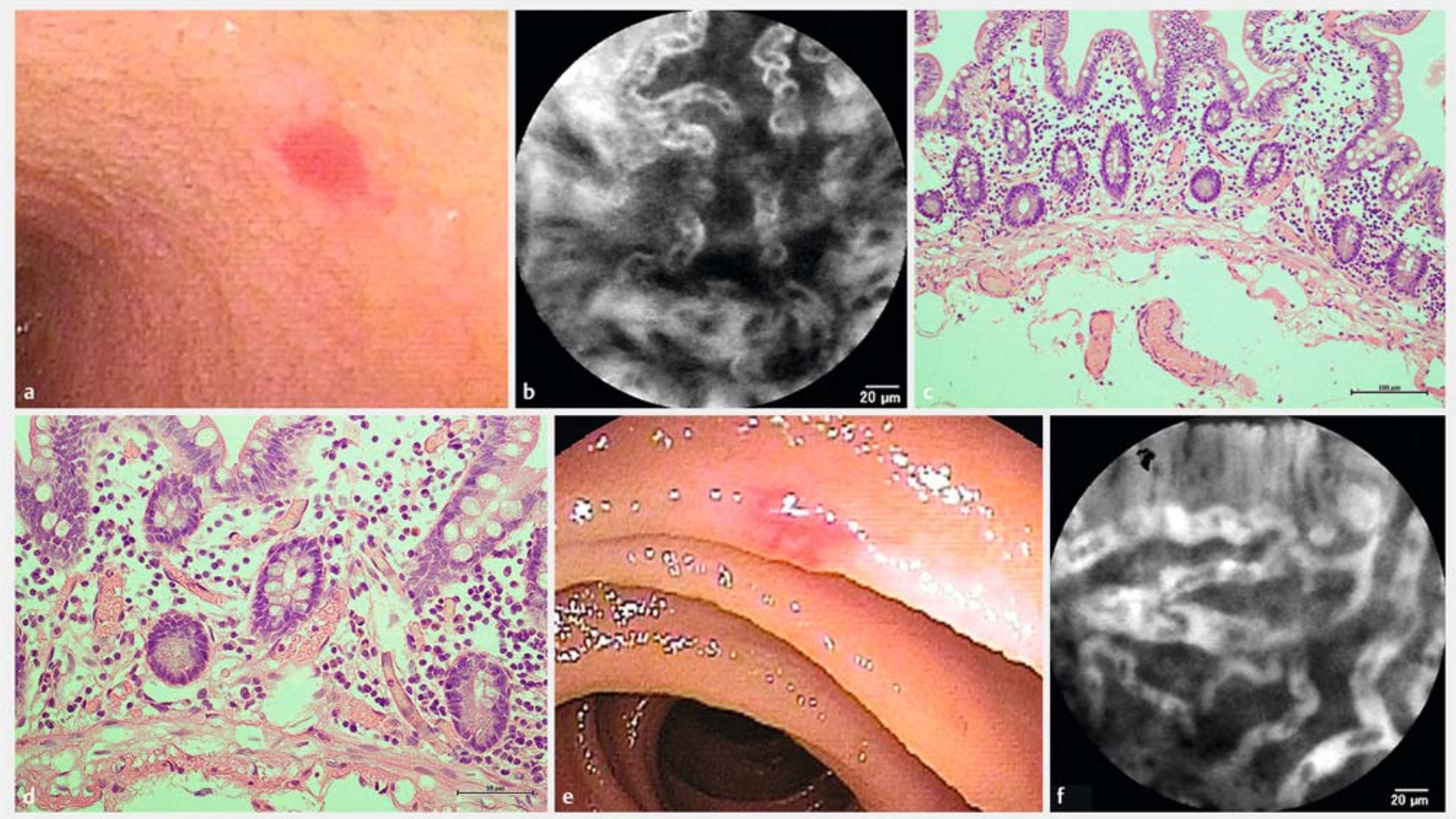

- Fig. 5 Arteriovenous malformation. a Enteroscopic view of hereditary hemorrhagic telangiectasia in the ileum (Patient \#11). b A probebased confocal laser endomicroscopic image shows a micro-arteriovenous malformation. $\mathbf{c}$ H\&E staining in an endoscopic mucosal resection specimen observed in $\mathbf{a}$ and $\mathbf{b}$ with a magnification of $\times 200$. $\mathbf{d}$ H\&E staining in an endoscopic mucosal resection specimen observed in $\mathbf{a}$ and b with a magnification of $\times 400$. e Enteroscopic view of a solitary angiodysplasia in the jejunum (Patient \#12). $\mathbf{f}$ A probe-based confocal laser endomicroscopic image shows a micro-arteriovenous malformation.
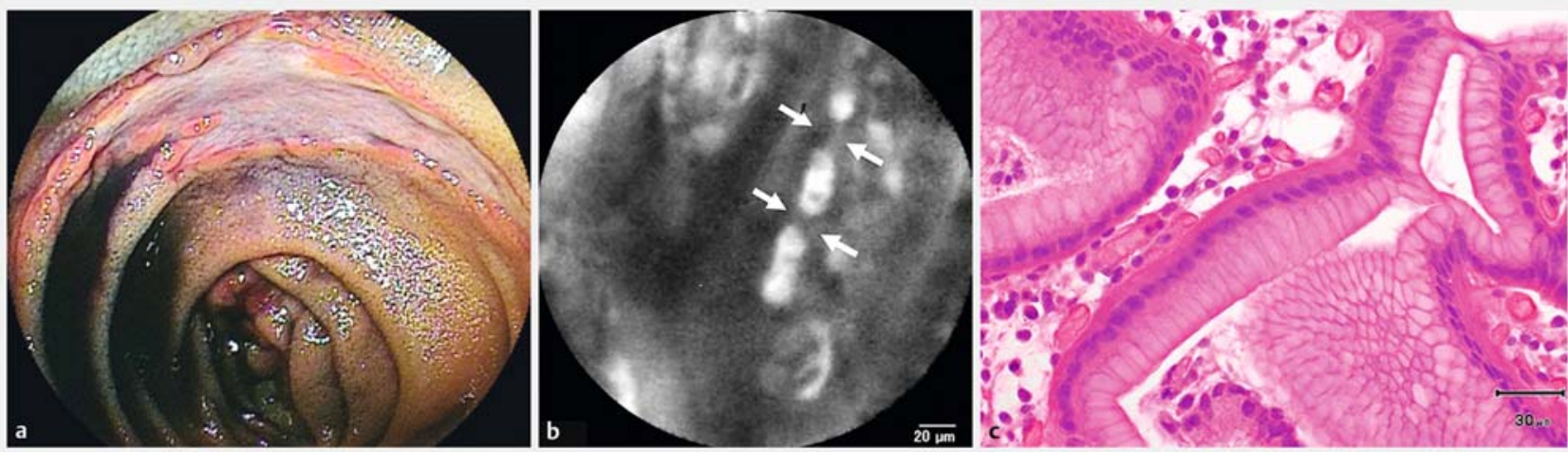

- Fig. 6 IgA vasculitis (Patient \#13). a Enteroscopic view in the jejunum. b A probe-based confocal laser endomicroscopic image. Arrows indicate segmental strictures of a capillary vessel, similar to "a string of sausages". c H\&E staining in a biopsy specimen shows leukocytoclastic vasculitis with a magnification of $\times 400$.

lymphoma cells were observed pathologically, but pCLE images showed homogenous tumor-like cells with dilated capillary vessels, which were presumably inflammatory cells caused by complicating cytomegalovirus infection. In patient \#32 with intestinal lymphangiectasia and with protein-losing enteropathy, lymphatic vessels were seldom observed with $\mathrm{PCLE}$ examination because they were predominantly subserosal lymphangiectasia type. In patient \#33 with Meckel's diverticulum, an aberrant gastric mucosa was observed pathologically, but was not observed with pCLE examination.

\section{Adverse events}

There were no complications associated with DBE, pCLE, and intravenous injection of fluorescein including bleeding, perforation, aspiration pneumonia, pancreatitis, and anaphylaxis. 

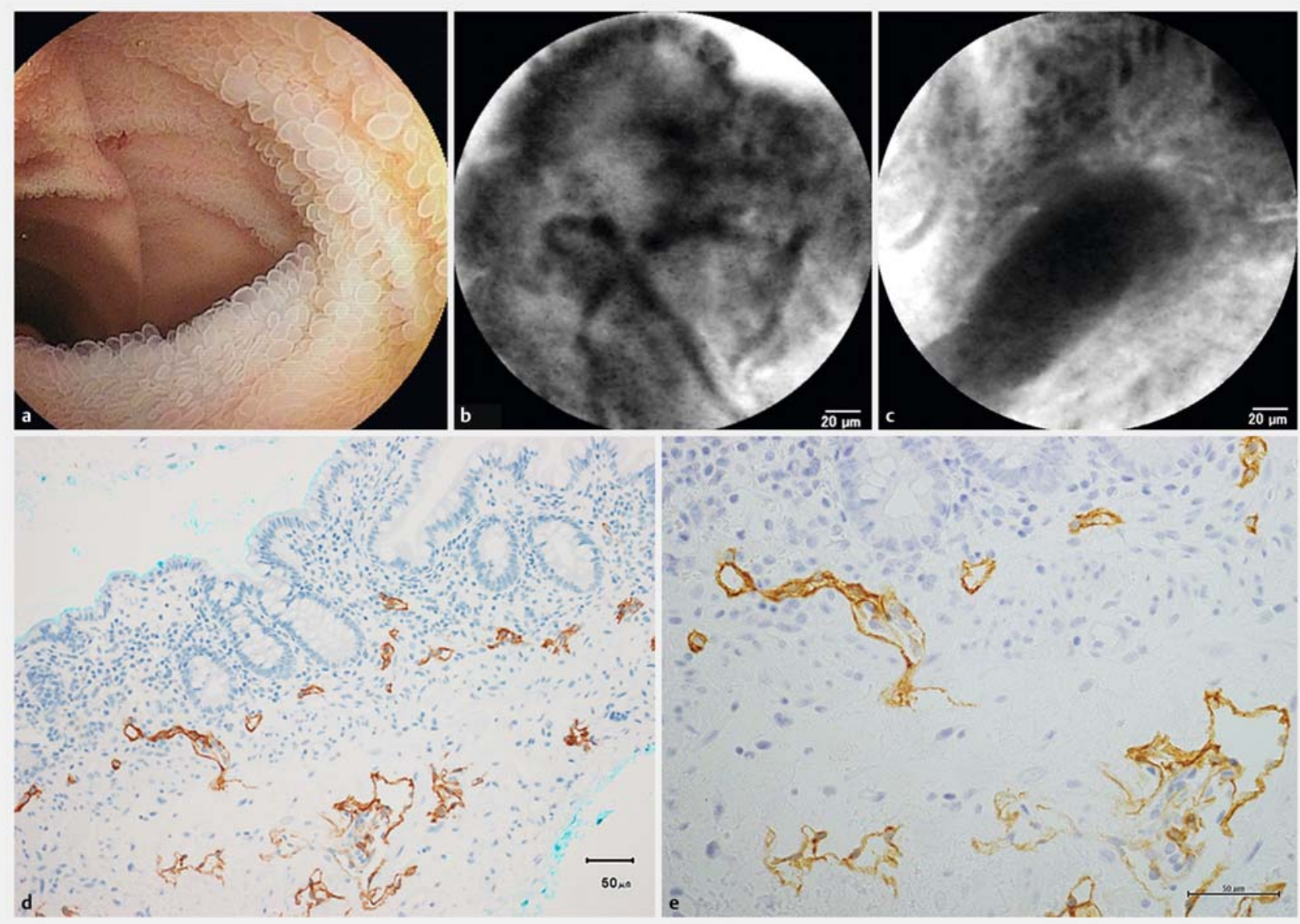

- Fig. 7 Intestinal lymphangiectasia, non-white type with protein-losing enteropathy (Patient \#30). a Enteroscopic view with small round villi in the ileum. $\mathbf{b}$ A probe-based confocal laser endomicroscopic image on the surface of the villus with unenhanced lymphatic vessels. $\mathbf{c}$ A probebased confocal laser endomicroscopic image deep in the mucosal layer with an unenhanced lymphatic duct. $\mathbf{d}$ Immunohistochemical staining with D2-40 antibodies in a biopsy specimen. Magnification $\times 200$. e Immunohistochemical staining with D2-40 antibodies in a biopsy specimen. Magnification $\times 400$.

\section{Discussion}

As shown in the present study, CLE was able to visualize in detail the capillary and lymphatic vessels and the subsurface architecture of the mucosa in real time [6], which was not possible with ordinary formalin-fixed paraffin-embedded tissue sections. The present preliminary comparison between immunostaining with CD34 antibodies and pCLE suggested shrinkage of capillary vessels caused by the interruption of blood flow with endoscopic biopsy or resection. In comparisons between immunostaining with D2-40 antibodies and PCLE, however, histology showed that the inner diameters of the lymphatic vessels were larger than in the pCLE images, presumably because the lymphatic vessels were not shrunk immediately by the interruption of lymph flow, and/or because deep mucosal lymphatic vessels were being measured. Therefore, $\mathrm{pCLE}$ is a valuable tool for examining in vivo capillary and lymphatic changes in various pathologies.

In malignant lymphoma, aggressive lymphomas, such as diffuse large B cell lymphoma, showed larger irregular capillary vessels than indolent B cell lymphomas, such as follicular lymphoma. Metastasis from cervical cancer also showed this, as shown in > Fig. 4. Santi et al. demonstrated that the microvascular calibers of resected specimens were significantly larger in submucosally invasive esophageal cancer than in intramucosal cancer [7]. Deb et al. reported that histological grades of brain tumors positively correlated with microvessel calibers in surgical specimens [8]. In tumors, irregular dilated capillary vessels may be indicative of the malignancy grade. We have also proposed a "soccer ball-like pattern" constituting homogenous dark cells packed with polygonal, slightly narrow and stretched capillary vessels, which was characterized by a pCLE image of a malignant lymphoma follicle. This characteristic pattern might be indicative of endoscopic assessment after treatment.

In vascular diseases, hereditary hemorrhagic telangiectasia (HHT) and solitary angiodysplasia showed numerous anastomoses of different calibers of capillary vessels. Meining et al. showed that the former version of pCLE detected a dilated sidled blood vessel with moving erythrocytes in real time, but not anastomosis due to poor resolution in gastric angiodyspla- 
sia [9]. To our knowledge, this is the first study to demonstrate in vivo blood vessel anastomosis in intestinal vascular malformation. In small-bowel ulcers associated with IgA vasculitis, "a string of sausages" pattern characterized by segmental stenosis of capillary vessels was observed, possibly leading to ischemic changes. Patient \#13 was easily diagnosed as having IgA vasculitis because he had the complication of prior purpura in his legs. Abdominal symptoms, however, can precede the onset of the characteristic purpuric rash in $14-36 \%$ of patients [10]. In such cases, pCLE may be useful for diagnosing vasculitis-associated ulceration because leukocytoclastic vasculitis is considered pathologically difficult to detect [11]. Chronic nonspecific multiple ulcers of the small intestine (CNSU) is a rare entity characterized by long-standing protein-losing enteropathy and iron-deficiency anemia from occult bleeding, reported chiefly in Japan, and recently published as an autosomal recessive inherited enteropathy caused by mutations in the SLCO2A1 gene, encoding a prostaglandin transporter [12-15]. The present study found the distinctive angulated stenosis of capillary vessels beneath the epithelium of the villi and cell gaps (data not shown), which may lead to the pathogenesis of multiple ulceration and protein loss.

Another notable finding of the present study was in vivo visualization of lymphatic vessels in small-bowel pathology. In intestinal lymphangiectasia with protein-losing enteropathy, lymphatic blockage can occur at different sites, and lymphangiectasia can be present in the lamina propria only, whereas marked dilation of the lymphatics can occur in the subserosa and mesentery, with minimal changes in the lamina propria [16]. We proposed a classification of intestinal lymphangiectasia into white villi type (the former lamina propria dominant type) and non-white villi type (the latter deeper layer dominant type) according to the enteroscopic findings, which clustered its manifestations and severity. Enteroscopically, the non-white villi type showed that apparently normal, but under more detailed observation, low and round villi with a normal color, were diffused [17]. In two of three patients with non-white villi type intestinal lymphangiectasia, pCLE was able to detect lymphangiectasia, while detection did not occur in the remaining patient who was diagnosed only by autopsy, showing predominantly subserosal lymphangiectasia accompanied by marked fibrosis in the submucosal layer (data not shown). In all three patients, numerous cell gaps were also detected by pCLE, probably leading to protein-losing enteropathy.

In inflammatory diseases, including Crohn's disease and CNSU, interestingly, the inner diameters of capillary vessels beneath the epithelium were smaller. Kiesslich et al. found increased epithelial gaps and fluorescein leakage in the small intestine of patients with inflammatory bowel disease, which was associated with subsequent relapse [18]. In this study, the same pCLE findings were observed depending on the enteroscopic findings. Narrowing of capillary vessels beneath the epithelial cells observed in this study might be ascribed to fluorescein leakage in the lamina propria and out of the epithelium.

Taken together, this in vivo histologic imaging has several clinical advantages over biopsy samples followed by a conventional pathology processes, including fixation, paraffin embed- ding, and staining steps: 1) to obtain live microscopic images, especially of capillary and lymphatic vessels, 2) to obtain realtime microscopic images of endoscopically ambiguous non-apparent lesions without time loss [19], 3) to obtain the functional properties including vascular permeability and cell shedding of fluorescence, 4) to benefit patients on antithrombotics, with bleeding tendencies, or with a condition for which taking pathological samples is contraindicated or not recommended, and 5) to obtain molecular imaging with administration of fluorescence-conjugated antibodies [20].

This study has some limitations, i.e. a small sample size and retrospective approach. Further larger-scale and prospective studies are needed to clarify the reliability of the above mentioned pCLE findings for diagnosing various small-bowel diseases. If our ongoing optical histologic findings are corroborated, it will help with rapid diagnosis and therapy selection. In conclusion, pCLE is safe and useful for the analysis of abnormalities of capillary and lymphatic vessels and the epithelium in various small-bowel diseases.

\section{Competing interests}

The authors report no potential conflicts of interest.

\section{References}

[1] Wallace MB, Fockens P. Probe-based confocal laser endomicroscopy. Gastroenterology 2009; 136: 1509-1513

[2] Wang KK, Carr-Locke DL, Singh SK et al. Use of probe-based confocal laser endomicroscopy ( $\mathrm{pCLE}$ ) in gastrointestinal applications. A consensus report based on clinical evidence. United Eur Gastroenterol ] 2015; $3: 230-254$

[3] Miehlke S, Morgner A, Aust D et al. Probe-based confocal laser endomicroscopy in double balloon enteroscopy. Z Gastroenterol 2011; 49: $1529-1534$

[4] Ohmiya N, Taguchi A, Shirai K et al. Endoscopic resection of Peutz-Jeghers polyps throughout the small intestine at double-balloon enteroscopy without laparotomy. Gastrointest Endosc 2005; 61: 140 147

[5] Arakawa D, Ohmiya N, Nakamura M et al. Outcome after enteroscopy for patients with obscure GI bleeding: diagnostic comparison between double-balloon endoscopy and videocapsule endoscopy. Gastrointest Endosc 2009; 69: 866-874

[6] Nguyen DL, Lee JG, Parekh NK et al. The current and future role of endomicroscopy in the management of inflammatory bowel disease. Ann Gastroenterol 2015; 28: 331 - 336

[7] Santi EG, Inoue H, Ikeda H et al. Microvascular caliber changes in intramucosal and submucosally invasive esophageal cancer. Endoscopy 2013; 45: 585-588

[8] Deb P, Boruah D, Dutta V. Morphometric study of microvessels in primary CNS tumors and its correlation with tumor types and grade. Microvasc Res 2012; 84: 34-43

[9] Meining A, Bajbouj M, Schmid RM. Confocal fluorescence microscopy for detection of gastric angiodysplasia. Endoscopy 2007; 39: (Suppl. 01): E145

[10] Choong CK, Beasley SW. Intra-abdominal manifestations of HenochSchonlein purpura. J Paediatr Child Health 1998; 34: 405 -409 
[11] Geboes K, Dalle I. Vasculitis and the gastrointestinal tract. Acta Gastroenterol Belg 2002; 65: 204-212

[12] Matsumoto T, lida M, Matsui T et al. Non-specific multiple ulcers of the small intestine unrelated to non-steroidal anti-inflammatory drugs. J Clin Pathol 2004; 57: 1145 - 1150

[13] Matsumoto T, Nakamura S, Esaki M et al. Endoscopic features of chronic nonspecific multiple ulcers of the small intestine: comparison with nonsteroidal anti-inflammatory drug-induced enteropathy. Dig Dis Sci 2006; 51: 1357-1363

[14] Takenaka H, Ohmiya N, Hirooka Y et al. Endoscopic and imaging findings in protein-losing enteropathy. J Clin Gastroenterol 2012; 46: $575-580$

[15] Umeno J, Hisamatsu T, Esaki M et al. A hereditary enteropathy caused by mutations in the SLCO2A1 gene, encoding a prostaglandin transporter. PLoS Genet 2015; 11: e1005581
[16] Vardy PA, Lebenthal E, Shwachman H. Intestinal lymphangiectasia: a reappraisal. Pediatrics 1975; 55: 842-851

[17] Ohmiya N, Nakamura M, Yamamura T et al. Classification of intestinal lymphangiectasia with protein-losing enteropathy: white villi type and non-white villi type. Digestion 2014; 90: 155-166

[18] Kiesslich R, Duckworth CA, Moussata D et al. Local barrier dysfunction identified by confocal laser endomicroscopy predicts relapse in inflammatory bowel disease. Gut 2012; 61: 1146 - 1153

[19] Canto MI, Anandasabapathy S, Brugge W et al. In vivo endomicroscopy improves detection of Barrett's esophagus-related neoplasia: a multicenter international randomized controlled trial (with video). Gastrointest Endosc 2014; 79: 211 - 221

[20] Atreya R, Neumann H, Neufert $C$ et al. In vivo imaging using fluorescent antibodies to tumor necrosis factor predicts therapeutic response in Crohn's disease. Nat Med 2014; 20: 313 - 318 